\title{
WEB ENGINEERING APPROACHES FOR REQUIREMENT ANALYSIS A Systematic Literature Review
}

\author{
Jose Alfonso Aguilar, Irene Garrigós, Jose-Norberto Mazón and Juan Trujillo \\ Lucentia Research Group, University of Alicante, Spain \\ jaac12@alu.ua.es, igarrigos@dlsi.ua.es,jnmazon@dlsi.ua.es,jtrujillo@dlsi.ua.es
}

Keywords: Requirement Analysis, Web Engineering, Systematic Review.

\begin{abstract}
Web engineering software development is facing continuous changes in technology implementation. This involves analysts, developers and designers to provide extra effort in the design and maintenance of Web applications in order to adapt them to changes in requirements and implementation technologies. In this paper, a systematic review is presented in order to obtain, in a formal way, the current state-of-the-art about approaches for modeling, analysis and specification of Web engineering requirements, supported with a formal and well defined strategy.
\end{abstract}

\section{INTRODUCTION}

One of the most important factors of success in the software development is the elicitation, management, and analysis of requirements. This is especially true in Web engineering due to the heterogeneous audience of the Web, which may lead to websites difficult to comprehend by visitors and complex to maintain by designers (Garrigós, Mazón et al. 2009). Importantly, Web applications have certain characteristics that make them different from traditional software or information systems such as the amount of information they offer (content), the access to the different scenarios where they offer this information (navigational) and how providing this information to the user or the groups of users (functionality) of the website. These unique characteristics of Web applications enforced new Web engineering methodologies to cope with those new requirements and Web developers need to adopt it.

The goal of this work is to make a comprehensive review and synthesis of the current state-of-the-art in the literature related to the specification of Web engineering requirements with the purpose of showing gaps in current research and shed light on potential future research lines. In this paper, we expect to study methods and techniques that propose the specification of Web requirements and bring some kind of support to generate several models of the website from the requirements.

The main objective of this work has been achieved through a systematic review using the approach of Kitchenham (Kitchenham 2004) and taking into account Mark Staples experiences (Staples and Niazi 2007).

\section{BACKGROUND}

Web Engineering (WE) is a branch of Software Engineering (SE) that defines techniques, processes and specific models for the Web environment. WE has a sub-discipline that refers to the phase in application development in which requirements of different stakeholders are gathered and processed, resulting in a requirements specification, this phase is called Requirements Engineering (RE) (Almeida, van Eck et al. 2006).

In this sense, one problem associated with RE is that requirements should be fully completed. To this aim, it is necessary that users and stakeholders can see that it has completed the transformation of their requirements in the final work product and be able to distinguish which one belongs to a certain requirement, this will help determining which requirements will be impacted due to the modification of a work product or vice versa. Furthermore, requirements engineering needs to ensure Requirements Traceability (RT), RT refers to the ability to describe and follow the life of a 
requirement, in both a forwards and backwards direction (Gotel and Finkelstein 1994). Forward traceability concerns to follow the requirement to its final implementation. Backward traceability refers to follow the work product to its source requirement (i.e. the associated requirement that originate it).

Recently, the Model-Driven Development (MDD) has become an alternative to solve the problems associated with both SE and WE. MDD was proposed by the OMG (Object Management Group). The key idea is that if the software development is guided by models that represent the final software to develop, some benefits will be obtained in some aspects like functionality, interoperability and maintenance.

\section{METHODOLOGY}

The interest of developing a systematic review starts with the need summarizing existing information about the formal processes for requirements analysis (RA) and specification of Web engineering through modeling and their traceability. This is done with the purpose of establishing more general conclusions revealing the advantages and disadvantages of each approach and to establishing a starting point for future research. To do this, we consider the following research questions:

1. Which Web engineering approaches do exist that cover the requirements analysis in Web engineering and which support requirements traceability?

2. Which are the techniques proposed in Web engineering approaches for requirements analysis and traceability?

3. Which tools have been applied in Web engineering approaches to requirements analysis and which with integrated support for traceability?

4. Which shortcomings have been detected until now in Web engineering approaches in relation to requirements analysis and traceability?

Once we have the research questions, the next step was selecting the digital resources for the search. The resources available for conducting the investigation were: eJournals (ACM, IEEE, Science Direct), Digital library of scientific literature (DBLP Computer Science Bibliography), World Wide Web (Google Scholar). After having selected the search sources, the strings to use for the search were defined. Finally, 13 relevant papers were found in total resulting from the execution of the search strings and search protocol.

Once we have the primary studies, the next step is to extract and summarize the information answering the research questions. As a result, the following Web approaches are presented.

UWE (UML-based Web Engineering. It proposes interviews, questionnaires and checklists as appropriated techniques for the requirements capture (Escalona and Koch 2004). Regarding to the requirements specification, this phase is carried out by means of UML Profiles (Escalona and Koch 2007). This approach does not provide traceability support. With regard to the implementation, UWE has a plugin called MagicUWE to be used with the CASE tool MagicDraw (Busch and Koch 2009). It is worth to mention that in (Kroiss, Koch et al. 2009) the author proposes a set of plugins for Eclipse called UWE4JSF for the automatic generation of Web applications in JSF (JavaServer Faces) derived from UWE models.

Navigational Development Techniques (NDT). In requirements analysis phase NDT applies use case diagrams and a set of templates for requirements with a textual description (Escalona and Aragon 2008). Traceability is supported through a set of traceability matrices that keeps the relationship between a requirement and their respective artifact that satisfies it (Escalona, Mejías et al. 2004). Requirements analysis phase and traceability is supported by the NDT-Suite tool (NDT-Profile, NDT-Driver-Quality NDT) using profiles to work with the CASE tool Enterprise Architect. In (Escalona and Koch 2007), the authors of NDT and UWE have developed an UML profile for Web requirements called WebRE. In combination of both approaches, the conceptual models can be derived from requirements specification; the main drawback of this approach is the lack of tool support for model-to-model transformations.

Web Modeling Language (WebML). This is a method for designing websites that allow high-level modeling (Ceri, Fraternali et al. 2000). The requirements analysis phase is not described in detail, but in (WebML 2009) the author proposes the use of UML (Unified Modeling Language) using use case and activity diagrams for its specification. This approach does not have traceability support.

WSDM: Web Site Design Method. The initial two phases of this approach (Mission Statement and User Modeling) are responsible for managing requirements through techniques such as concept maps (of roles and activities) and the data dictionary for the definition of functional and security requirements (De Troyer and Leune 1998). The requirements in this approach are specified in a textual form. The lack of transformations between models and support for traceability as well as the lack of a prototype tool which supports the 
Table 1: Summary of methodological approaches.

\begin{tabular}{|c|c|c|c|c|c|}
\hline \multirow{2}{*}{ Approach } & \multirow{2}{*}{ Techniques } & \multirow{2}{*}{ Traceability Support } & \multicolumn{3}{|c|}{ Tools } \\
\cline { 4 - 6 } & & Yes & NDT-Tool & NDT-Tool & NDT-Suite \\
\hline NDT & Use cases, textual templates & No & WebRatio & No & No \\
\hline WebML & Use cases,activity diagrams & No & No & No & No \\
\hline WSDM & Concept maps, data dictionary & No & ArgoUWE & MagicUWE & No \\
\hline \multirow{2}{*}{ UWE } & Use cases, UML profiles & & & ArgoUWE & AGG \\
\hline OOWS & Use cases, Task diagrams, FRT & Yes & OlivaNova & OlivaNova & TaskTracer \\
\hline A-OOH & Use cases, $i^{*}$, UML profiles & No & VisualWade & Eclipse Plugin & \multirow{2}{*}{ No } \\
\hline
\end{tabular}

requirements specification demonstrates the limitations of this approach.

\section{OOWS: An Object-Oriented Approach for Web} Solutions Modeling. This approach provides a gathering requirements phase through a series of strategies that implement FRT (Function Refinement Tree), use cases and a set of diagrams (tasks, task specification and data description) for navigation requirements. This approach has traceability support (Valderas and Pelechano 2009), this is done through a set of transformations rules defined by graph theory. In regard to tool support, this approach has an environment called OOWS-Suite (Valverde, Valderas et al. 2007), which is integrated with the OlivaNova tool to provide support for requirements gathering phase. For the traceability tool support, this approach uses two tools, the first one is the open source tool called AGG (Attributed Graph Grammar System) and the second is TaskTracer developed by the authors to generate traceability reports.

A-OOH (Adaptive Object Oriented Hypermedia). It is an extension of the $\mathrm{OOH}$ modeling method (Object Oriented Hypermedia) addressed to the user. The techniques used in this approach for the requirements specification are the $i^{*}$ framework and UML-Profiles. The requirements model is specified by the designer using i* models, specifically the SR (Strategic Relationship) model and the SD (Strategic Dependency) model. Next, the conceptual models are generated by means of QVT transformations, thus are considering the goals and needs of stakeholders that will meet the expectations of users thereby reducing errors that may appear on the final implementation. The traceability between the models generated by this approach could be derived from the transformations rules. The requirements specification phase in A-OOH has been implemented using the Eclipse development platform (Garrigós, Mazón et al. 2009).
Next, a brief analysis about the approaches described above is presented, to facilitate this analysis Table 1 is shown.

Table 1 shows a tendency towards the application of UML profiles as a technique used by the approaches studied in this systematic review and the persistence of other one, the use cases. Moreover, although traceability is a very important success factor for software engineering, a common problem in Model-Driven Web development processes is that most of the approaches lack support for traceability (except NDT and OOWS).

Except WSDM, each approach has a tool to support it. In the requirements phase, only NDT, UWE, OOWS and A-OOH have a tool support. In terms of traceability, the two approaches that have implemented traceability have a tool support. It is worth noting that OOWS combines two tools to achieve this support.

In this context, the approaches explored in this systematic review do not consider the real user expectations of the website as well as stakeholders from an early stage of requirements analysis. A$\mathrm{OOH}$ is the exception, as it considers these expectations through the $\mathrm{i} *$ framework where requirements are modeled based in the user goals and objectives, thus avoiding the requirement specification in textual form (with the effort involved to do it this way) and the based on tasks (depending in most cases the analyst's experience).

Is worth mentioning the support offered by the approaches WSDM, NDT, UWE and WebML through its website, they offer examples, published papers and their respective tools for everyone who visits their website, except WSDM which only offers the download of published papers due to that is a tool that has proprietary licence. In the particular case of UWE and WebML is necessary to mention that in their website they have guided step by step 
examples to study and practice the developing of a Web application using their respective support tool. This confirms that these two approaches are mostly used in academic (university) projects.

Finally, MDD is applied successfully by several Web engineering methods. However, any approach has a fully guided methodology for the analysis of Web engineering requirements. To sum up, current situation of Web engineering approaches presents the following drawbacks: i) lack of traceability support, ii) RA phase without real user goals and needs; and iii) soundless tool support.

To solve the drawbacks mentioned above, we propose the development of a solution at conceptual modeling level using the approach A-OOH. With regard to the first drawback (lack of support for traceability), the solution will be implemented using Weaving Models in model-to-model transformations for traceability support. For the second one (RA phase without real user goals and needs), will be used the $i^{*}$ framework models for the requirements specification, the requirements model is specified by the designer using $i^{*}$ models, specifically the SR (Strategic Relationship) model and the SD (Strategic Dependency) model. Finally, these drawbacks will be supported by an open source tool.

\section{CONCLUSIONS}

This work presents the results obtained after carrying out a systematic review of literature whose aim was to make a comprehensive review and synthesis of the current state of the art in the literature related to modeling of Web engineering requirements. To do this, a total of 2813 papers published in literature and extracted from the most relevant scientific sources considered, of which 43 were eventually analyzed in depth, in accordance with the systematic review process adopted.

The results of this systematic review shows that Web engineering methods were not designed to address the design through the analysis of Web engineering requirements. Therefore our future work will be to prove the feasibility of applying the technologies previously-mentioned to propose a design approach guided by the analysis and traceability of requirements in Web engineering and supported by an open source tool.

\section{REFERENCES}

Almeida, J. P., van Eck, P. A. T. \& Iacob, M. E. 2006. Requirements traceability and transformation conformance in model-driven development. 10th IEEE International Enterprise Distributed Object Computing Conference (EDOC'06). Hong Kong: IEEE Computer Society Press.

Busch, M. \& Koch, N. 2009. MagicUWE: A CASE Tool Plugin for Modeling Web Applications. 9th International Conference on Web Engineering. San Sebastian, Spain: Springer.

Ceri, S., Fraternali, P. \& Bongio, A. 2000. Web Modeling Language (WebML): a modeling language for designing Web sites. Computer Networks, 33, 137157.

De Troyer, O. M. F. \& Leune, C. J. 1998. WSDM: a user centered design method for Web sites. Computer Networks and ISDN Systems, 30, 85-94.

Escalona, M. \& Koch, N. 2004. Requirements engineering for Web Applications: a comparative study. Journal of Web Engineering, 2, 193-212.

Escalona, M., Mejías, M. \& Torres, J. Year. Developing systems with NDT \& NDT-Tool. In: 13th Information System Development, 2004 Lithuania. 149-59.

Escalona, M. J. \& Aragon, G. 2008. NDT. A ModelDriven Approach for Web Requirements. Software Engineering, IEEE Transactions on, 34, 377-390.

Escalona, M. J. \& Koch, N. 2007. Metamodeling the Requirements of Web Systems. Lecture Notes in Business Information Processing. Vol. 1. 2007. Pag. 267-282

Garrigós, I., Mazón, J.-N. \& Trujillo, J. 2009. A Requirement Analysis Approach for Using i* in Web Engineering. 9th International Conference on Web Engineering. San Sebastian, Spain: Springer-Verlag.

Gotel, O. C. Z. \& Finkelstein, C. W. Year. An analysis of the requirements traceability problem. In: Requirements Engineering, 1994., Proceedings of the First International Conference on, 1994. 94-101.

Kitchenham, B. 2004. Procedures for performing systematic reviews. Keele University and National ICT Australia Ltd, 1-28.

Kroiss, C., Koch, N. \& Knapp, A. 2009. UWE4JSF: A Model-Driven Generation Approach for Web Applications. Proceedings of the 9th International Conference on Web Engineering. San Sebastian, Spain: Springer-Verlag.

Staples, M. \& Niazi, M. 2007. Experiences using systematic review guidelines. J. Syst. Softw., 80, 14251437.

Valderas, P. \& Pelechano, V. 2009. Introducing requirements traceability support in model-driven development of web applications. Information and Software Technologies, 51, 749-768.

Valverde, F., Valderas, P. \& Fons, J. Year. OOWS Suite: Un Entorno de desarrollo para Aplicaciones Web basado en MDA. In: 11th International Database Engineering \& Applications Symposium, 2007 Banff, Canada.

WebML. 2009. WebML: The Web Modeling Language. Retrieved: July 2009, from http://www.webml.org. 